\title{
Architecture of a student training computer program for preparing professional outpatient consulting skills within an electronic medical records system during COVID-19 alertness situation
}

\author{
Sergey Bulatov ${ }^{1}$, Evgeni Magid ${ }^{2}$, Enzhe Kharisova ${ }^{1}$, Roman Lavrenov ${ }^{2}$, Vitaly Dudin ${ }^{1}$, Artur Khazetdinov $^{2}$ \\ ${ }^{1}$ Center of a practical skills, Kazan State Medical University, 49 Butlerov street, Kazan, Russian Federation \\ ${ }^{2}$ Laboratory of Intelligent Robotic Systems (LIRS), Intelligent Robotics Department, Institute for Information \\ Technology and Intelligent Systems, Kazan Federal University, 35 Kremlyovskaya street, Kazan, Russian Federation
}

\begin{abstract}
One of the important goals of a medical education in the context of the COVID-19 pandemic is a broad introduction of simulated-based teaching methods, which might include elements of robotics and artificial intelligence. We analyzed computer programs that are currently used for maintaining medical records of patients by various polyclinics of Kazan city. Based on these results, we summarized requirements for a training computer program that could provide students with medical records maintaining skills and developed program's preliminary architecture. Our main attention focused on modeling situations that are associated with pre-hospital stage processing of medical records for patients with suspected or confirmed COVID-19.
\end{abstract}

Keywords: medical education, computer program, COVID-19 infection

\section{Introduction}

The current epidemiological situation led to a transition of a higher medical education to distance learning ${ }^{1}$. While a main pedagogical emphasis moved to information technologies applications for educational process, including computer programs and artificial intelligence ${ }^{2,3}$, specifics of medical information systems (MIS) do not allow all students to master them independently. A preliminary training of students to work with computer MIS used in practical health care has become acute.

The COVID-19 pandemic, which already had infected almost 60 million people all around the world and took over 1.4 million lives just within less than a year, has been a contributing factor in this process as it changed a traditional doctor-patient relationship and replaced face-to-face communication with a remote monitoring of patients ${ }^{4}$. In local polyclinics, computer programs create electronic medical records (EMR) of patients, draw up sick lists, monitor laboratory tests, and ensure preservation of information about patients' treatment. In literature sources, the authors had found only isolated reports about special training of students to work EMRs and MIS 5 .

\section{Related work}

In medical universities of Russia, a special training of graduate students to work with MIS of a regional polyclinic is still experimental. Programs that are used in multi-specialty hospitals for inpatient patients might be employed as educational materials in universities, e.g., MIS "Aurora" for multi-specialty hospitals ${ }^{6}$ provides a training interface "UMS University" for individual tasks, with a separate entrance for students and teachers, and an access to a server via the Internet. The authors ${ }^{6}$ reported a positive impact of the training course on preparing students to work with a MIS.

In regional polyclinics of Kazan city various commercial MISs are used, including BARS.MED, ERP-system, 1C: Medicine (Registry), and VITACORE. These systems have centralized databases with a secure access for each user through standard operating environments (Microsoft, Linux) and a Web browser. Yet, application of training versions of these programs for student training was not reported.

In an international literature, an equivalent of a MIS is an EMR, which combines all types of MISs. A positive effect of using EMR in training of higher medical schools' students was established in $^{7,8,9}$ as an 
important part of an education, while students, in turn, reported that mastering EMR had a positive role in preparing them for a future profession ${ }^{10,11}$.

Today, a unified pedagogical approach of teaching students to work with EMR/MIS does not exist ${ }^{9}$. We believe, the reasons are related to a commercial nature of such products, which prevents their free distribution, as well as an absence of a single EMR/MIS that is required for an obligatory use by a local government. Therefore, in order to facilitate medical education, local teams of educators create unique training computer programs that simulate real MIS behavior to support the necessary training. Main characteristics of such training programs (in order to provide an acceptable level of simulation) are their similarity to existing commercial products, a capability to evaluate student's actions and to help him/her during a task execution. This paper presents a preliminary architecture and interface for the MIS training program for students of higher medical schools. As an example, we discuss a consultation of an outpatient patient in a polyclinic with signs of COVID19 infection.

\section{Program architecture and interfaces}

\subsection{Situation modeling}

Collecting information from a patient and conducting an objective examination of the patient comprise a preliminary stage of work. The stage begins from creating am instance for a patient database and filling out a corresponding EMR. A student manually enters his/her name, date and time of a consultation, and a patient's name to open active fields, which consist of complaints at the time of examination, medical history, patient's life history, and objective examination data (Fig. 1).

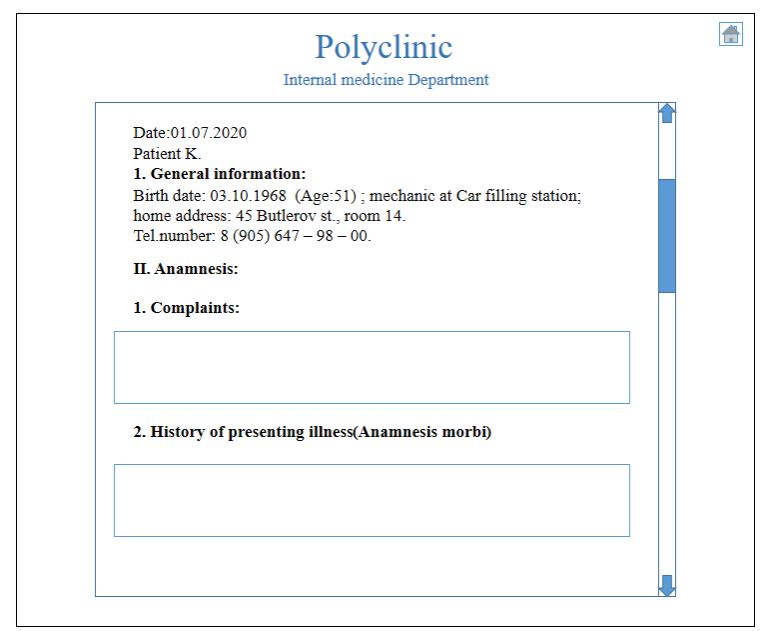

Fig. 1. Collecting information from a patient.

The stage ends with establishing a preliminary diagnosis and entering it in the corresponding field. The patient information is stored in the database and can be reused later by the student at the next stages.

\subsection{Analysis}

After making a preliminary diagnosis, in accordance with a treatment process algorithm, the patient should be assigned the necessary laboratory and instrumental assessment methods. It is necessary to select an optimal (strictly regulated by current standards) set of methods among proposed ones, which is performed by clicking interactive buttons on the right side of the screen. Next, the student can see all the results in a form of an actual data from a laboratory: a blood test, a CT scan of lungs, an electrocardiogram (Fig. 2).

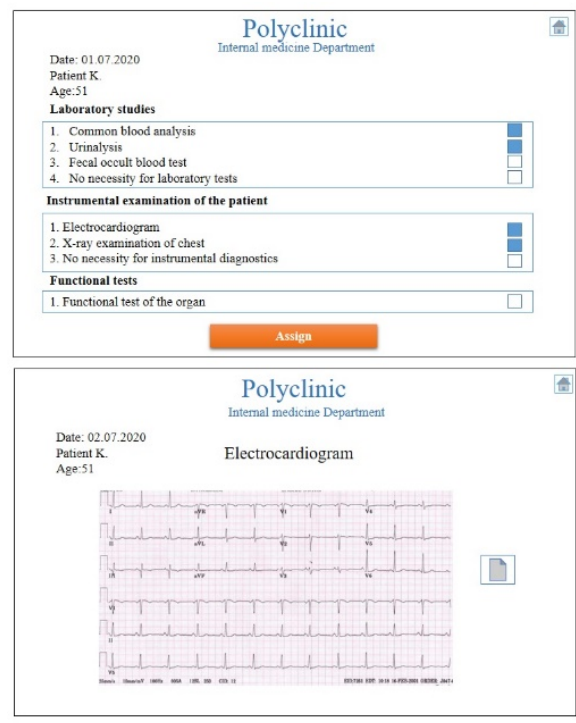

Fig. 2. Laboratory assessment methods.

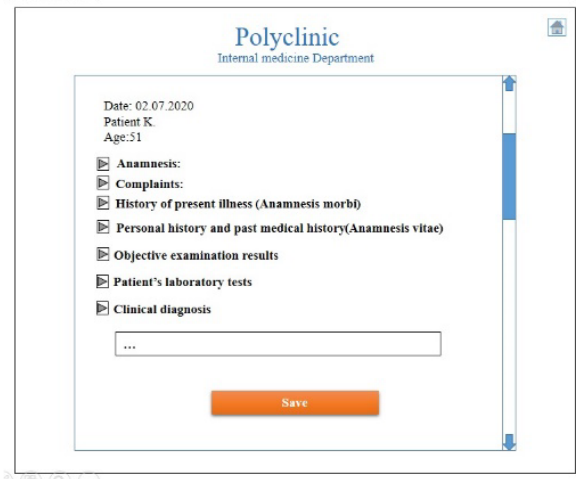

Fig. 3. Consultation and clinical diagnosis.

The student should identify existing deviations from normal ranges and mark them. The next step is to consult the patient with narrow profile specialist(s), which could be selected from a predefined list. In accordance with current medical standards, for certain diseases, these could be a cardiologist, an endocrinologist, a surgeon or other specialists (Fig. 3). The stage ends with creating a clinical diagnosis and recording it into a specially designated field. 


\subsection{Creating a treatment program}

On the next stage the student determines the patient's treatment plan. This is a creative process associated with a specialist qualification. An interface of this page has special fields where you the student can enter all details of the treatment. There is a field for prescribing medications, a recommended regimen, a diet, and other types of treatment (Fig. 4). At this stage, the student has an opportunity to refresh the patient information collected earlier and store it in the database.

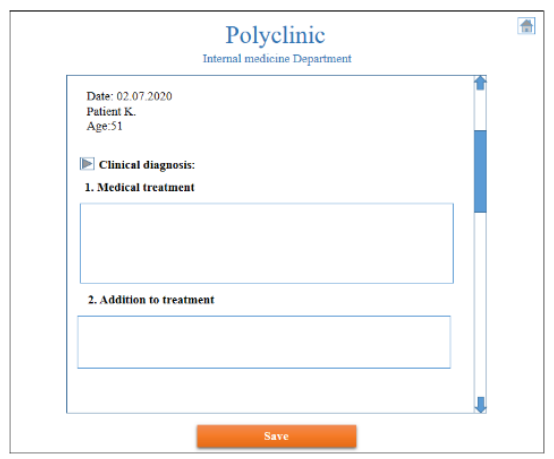

Fig. 4. Treatment strategy.

\subsection{Assessment}

The student's actions could be assessed by two methods: automatic (conducted by the program) and expert (conducted manually by a teacher). Based on the prescribed algorithm, the program evaluates a completeness of the patient's examination. If the patient information contains empty spaces, the program will require to complete this work.

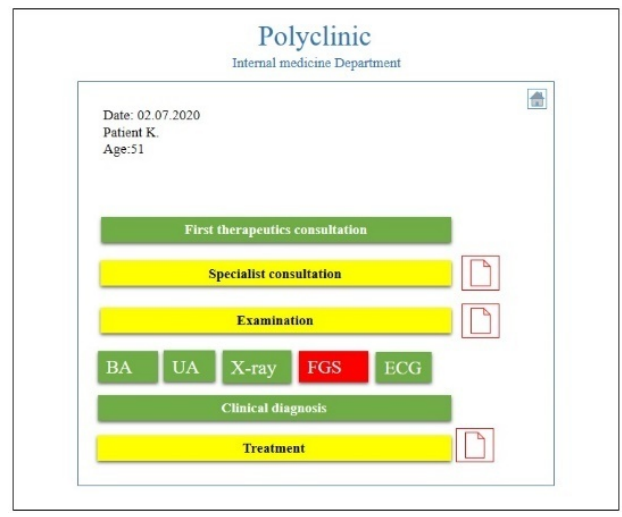

Fig. 5. Mistakes of a student's work.

The program uses color indicators (Fig. 5) as follows. Red color indicates an error, yellow indicates deviations from accepted standards, and green indicates the correct actions. The program allows students to improve knowledge using the interactive buttons that appear near the yellow and red fields. After pressing the button, a student can visit a library in the database and read regulatory documents and standards. A final assessment of the student's work is carried out by the teacher during an individual discussion.

\subsection{Example}

Today, the problem of treating patients with COVID-19 symptoms at home is of a great social significance. Every graduate medical student must study not only the main symptoms of this disease and know the treatment program, but also take into account nuances of monitoring a potential COVID-19 carrier in an outpatient setting. One of the goals of the program is to combine disparate information (patient data, proposed treatment and additional literature) within a single block to allow transforming theoretical knowledge into practical skills.

We constructed the following example case. The student plays a role of a clinic's entrance department doctor. Patient K., 51 years old, came with complaints of a high fever, a cough, and a general weakness. The student's task is to create an EMR for the patient and determine a medical treatment plan. The program focuses the student's attention on the most typical symptoms of COVID-19. In the "patient's complaints" field main signs of the disease appear in a form of a list. The student should ask the patient more carefully for each symptom (Fig. 6). The preliminary diagnosis of COVID-19 should be confirmed by appropriate laboratory and instrumental assessment (Fig. 7, 8).

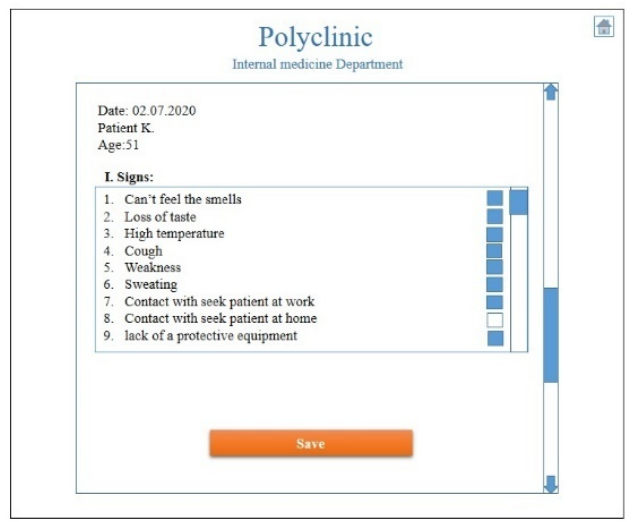

Fig. 6. Patient's complaints.

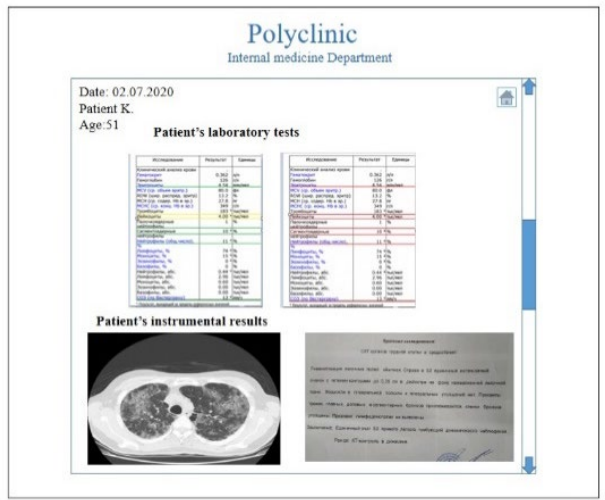

Fig. 7. Laboratory assessment results. 
The program generates a list of diagnostic tests that should be used for patients with suspected COVID-19. Next, the student should identify deviations from the normal ranges in laboratory assessment results of the patient. A correct decision is illustrated on the screen with a green background. Errors are marked in red, and incomplete solutions are marked in yellow. In this situation, a special symbol, which appears on the screen, recommends visiting the "library" in the database and studying the standards for laboratory and instrumental assessment of patients with suspected COVID-19. After successfully completing the first two stages and making a clinical diagnosis, the student is asked to make a comprehensive treatment plan for a suspected COVID19 carrier at home (Fig. 8).

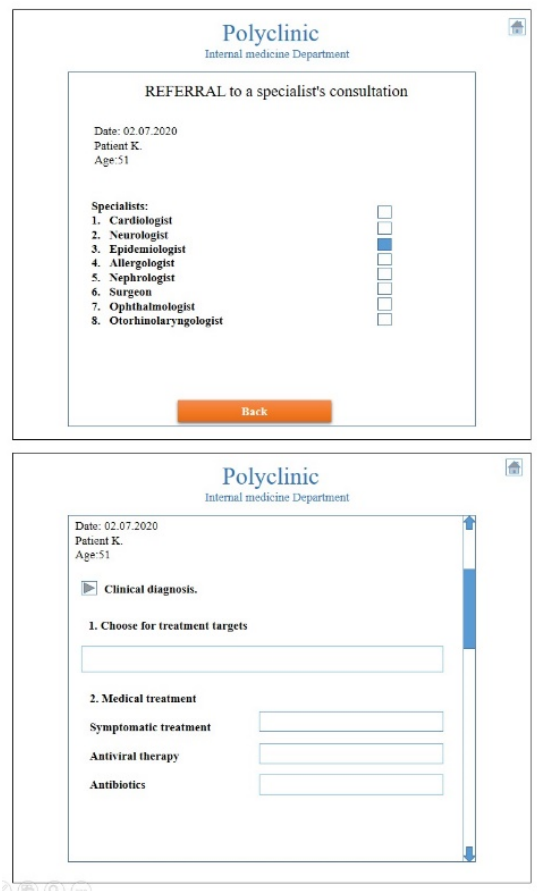

Fig. 8. Final steps to create a clinical diagnosis.

\section{Conclusions}

The paper presented a preliminary architecture and an interface for the MIS training program for students of higher medical schools. The program simulates main stages of a polyclinic doctor work in monitoring and treating patients at a pre-hospital stage, which opens up opportunities for independent and distance education. It allows creating EMRs and using a database with regulatory documents for outpatients' treatment. The proposed interface is similar to commercial software products currently used in polyclinics of Kazan city, Russia. The program develops practical skills of medical students in modern approach of EMR processing using artificial intelligence-based support for evaluation of outpatient's conditions, diagnosing a disease and selecting its treatment, including a special case of potentially infected with COVID-19 patients.

\section{References}

1. L. S. Schneiderand, M. L. Council (eds.), Distance learning in the era of COVID-19, Arch. Dermatol. Res., 2020, pp. 1-2.

2. S. Shah, S.Diwan, L. Kohan, D. Rosenblum, C. Gharibo, A. Soin, A. Sulindro, Q. Nguyen and D. A Provenzano, The technological impact of COVID-19 on the future of education and health care delivery, Pain Physician, 23(4S), 2020, pp. 367-380.

3. A. Sagitov, H. Li, N. Schiefermeier-Mach and E. Magid, Extending Gazebo simulator for surgical robotics: tissue and suture modeling. In International Conference on Artificial Life and Robotics, 2019, pp. 124-127.

4. H. Lal, D. K. Sharma, M. K. Patralekh, V. K. Jain and L. Maini, Outpatient department practices in orthopaedics amidst COVID-19: The evolving model, J. Clin. Orthop. Trauma, 11(4), 2020, pp.700-712.

5. M. Samadbeik, F.Fatehi, M.Braunstein, B. Barry, M.Saremian, F.Kalhor and S. Edirippulige, Education and Training on Electronic Medical Records (EMRs) for health care professionals and students: A Scoping Review, Int. J. Med. Inform., 142:104238, 2020, pp. 1-10.

6. S. I. Karas, I. O. Korneva, M. B. Arzhanik, O. L. Semenova, E. V. Chernikova, O. V. Urneva and A. Y. Grechishnikova, The role and prospects of information and communication technologies in developing of physician competencies, Doctor and information technology (IDMZ), №4, 2018, pp.46-58.

7. N. M. Pageler, C. P. Friedman and C. A. Longhurst, Refocusing medical education in the EMR era, JAMA, 310(21), pp. 2249-2250.

8. D. R. Cheng, T. Scodellaro, W. Uahwatanasaku and M. South, An Electronic medical record in pediatric medical education: survey of medical students' expectations and experiences, Appl. Clin. Inform, 9(4), 2018, pp. 809-816.

9. A. Rajaram, Z. Hickey, N. Patel, J. Newbigging and B. Wolfrom, Training medical students and residents in the use of electronic health records: a systematic review of the literature, J. Am. Med. Inform. Assoc., 27(1), 2020, pp. 175180.

10. C. Mountain, R. Redd, C. O'Leary-Kelly and K. Giles, Electronic medical record in the simulation hospital: does it improve accuracy in charting vital signs, intake, and output?, Comput. Inform. Nurs., 33(4), 2015, pp. 166-71.

11. C. M. Yuan, D. J Little, E. S Marks, M. A Watson, R. Raghavan and R. Nee, The electronic medical record and nephrology fellowship education in the united states: an opinion survey, Clin. J. Am. Soc. Nephrol., 15(7), pp. 949-956. 